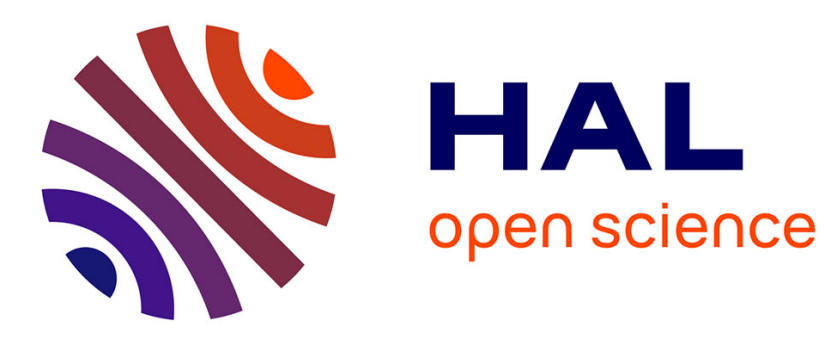

\title{
Etude d'un conjoncteur-disjoncteur à vide pour impulsion de courant intense
}

G. Bauville, A. Delmas, N. Haddad

\section{To cite this version:}

G. Bauville, A. Delmas, N. Haddad. Etude d'un conjoncteur-disjoncteur à vide pour impulsion de courant intense. Revue de Physique Appliquée, 1990, 25 (2), pp.199-207. 10.1051/rphysap:01990002502019900 . jpa-00246179

\section{HAL Id: jpa-00246179 \\ https://hal.science/jpa-00246179}

Submitted on 1 Jan 1990

HAL is a multi-disciplinary open access archive for the deposit and dissemination of scientific research documents, whether they are published or not. The documents may come from teaching and research institutions in France or abroad, or from public or private research centers.
L'archive ouverte pluridisciplinaire HAL, est destinée au dépôt et à la diffusion de documents scientifiques de niveau recherche, publiés ou non, émanant des établissements d'enseignement et de recherche français ou étrangers, des laboratoires publics ou privés. 


\title{
Etude d'un conjoncteur-disjoncteur à vide pour impulsion de courant intense
}

\author{
G. Bauville, A. Delmas et N. Haddad \\ Laboratoire d'Electrotechnique des Universités de Paris 6 et 11, (UA 845), Bâtiment 214, 91405 Orsay Cedex, \\ France
}

(Reçu le 18 décembre 1988, révisé et accepté le 24 octobre 1989)

\begin{abstract}
Résumé. - L'article est relatif à l'étude des capacités de conduction et de réisolement d'un conjoncteurdisjoncteur à vide. Le temps de conduction est de $27 \mu$ s et le temps de réisolement imposé est inférieur à $100 \mu \mathrm{s}$. Nous avons mis en évidence l'influence des paramètres physiques et géométriques afin d'obtenir le régime d'arc diffus donnant accès aux meilleures performances de disjonction. Ainsi le pouvoir de coupure du prototype testé, a dépassé $10^{9} \mathrm{~V} . \mathrm{A}(36 \mathrm{kA}, 44 \mathrm{kV})$.
\end{abstract}

\begin{abstract}
The article is relative to the recovery phenomena and conduction capacities of a fast triggered vacuum switch. $27 \mu \mathrm{s}$ current pulses are used. Fixed recovery times are less than $100 \mu \mathrm{s}$. We study the influence of physical and geometrical parameters in order to obtain the arc diffuse mode which allows best switching performances. Thus a breaking capacity more than $10^{9} \mathrm{~V}$. A is obtained with a $36 \mathrm{kA}$ current pulse and $44 \mathrm{kV}$ recovery voltage.
\end{abstract}

\section{Introduction.}

Nombre de travaux $[1,2]$ ont montré, en électrotechnique impulsionnelle, tout l'intérêt des sources d'énergie à stockage cinétique (alternateur, générateur homopolaire, génératrice asynchrone sans fer...), devant celles à stockage électrostatique, tant sur le plan densité massique d'énergie, que sur le plan économique. Pour des applications telles que la propulsion électromagnétique, la fusion contrôlée ou le magnétoformage lourd, les niveaux d'énergies impliquées appellent l'utilisation de telles sources. Un obstacle majeur apparaît dès que l'on s'intéresse à la puissance massique du système lors de la délivrance de l'énergie [1]. Pour accroître notablement cette puissance, il est nécessaire d'envisager une opération intermédiaire, susceptible d'assurer le transfert rapide de l'énergie, de la bobine de stockage (associée à la génératrice) vers la charge avec un rendement élevé [2, 3]. Le Laboratoire d'Electrotechnique d'Orsay développe actuellement un concept de transfert inductif faisant appel à un jeu de commutations permettant une désactivation progressive des spires de la bobine de stockage. Les performances d'une telle opération sont directement liées à celles des interrupteurs utilisés. Pour des niveaux d'énergie de $10^{7} \mathrm{~J}$, les puissances des commutations doivent pouvoir atteindre les $10^{12} \mathrm{~V}$. A pour des temps de conduction de $10^{-5} \mathrm{~s}$ et les vitesses d'extinction du courant dépassent les $10^{10} \mathrm{~A} / \mathrm{s}$. La tension appliquée après la phase de conduction est de l'ordre de $100 \mathrm{kV}$ pour un temps de réisolement d'environ $10^{-5} \mathrm{~s}$. Nous notons que cette tension est de même polarité que le courant, contrairement à ce que l'on rencontre dans la quasitotalité des applications. Dans ce contexte, nous avons engagé une étude exploratoire sur la conjonction-disjonction sous vide qui paraît être particulièrement bien adaptée à ce type de commutation rapide.

Les résultats déjà obtenus [4] sont fort encourageants, mais ils ne nous avaient pas permis de définir les conditions d'accès au régime d'arc diffus. A fréquence industrielle $(50-60 \mathrm{~Hz})$ ce dernier correspond au fonctionnement optimal du disjoncteur [5, 6].

L'étude présentée ici a pour but de préciser l'obtention du régime d'arc diffus afin d'optimiser les performances du conjoncteur-disjoncteur. Nous considèrerons donc deux types de paramètres : d'une 
part les paramètres géométriques tels que diamètre des électrodes et distance inter-électrode et, d'autre part les paramètres physiques tels que la nature des électrodes et l'action d'un champ magnétique annexe.

\section{Montage expérimental.}

2.1 LE PROTOTYPE. - La rapidité de la conjonction et de la disjonction exclut le déplacement mécanique des électrodes. Ainsi nous avons choisi une structure de type éclateur que nous avons présenté sur la figure 1. C'est un système de révolution formé de deux électrodes fixes. L'établissement de l'arc principal est obtenu grâce à une décharge auxiliaire, de moins de $1 \mathrm{~J}$, entre la cathode et une électrode d'amorçage. La pression à l'intérieur de l'enceinte du disjoncteur est maintenue à $10^{-6}$ torr par une pompe à diffusion d'huile.

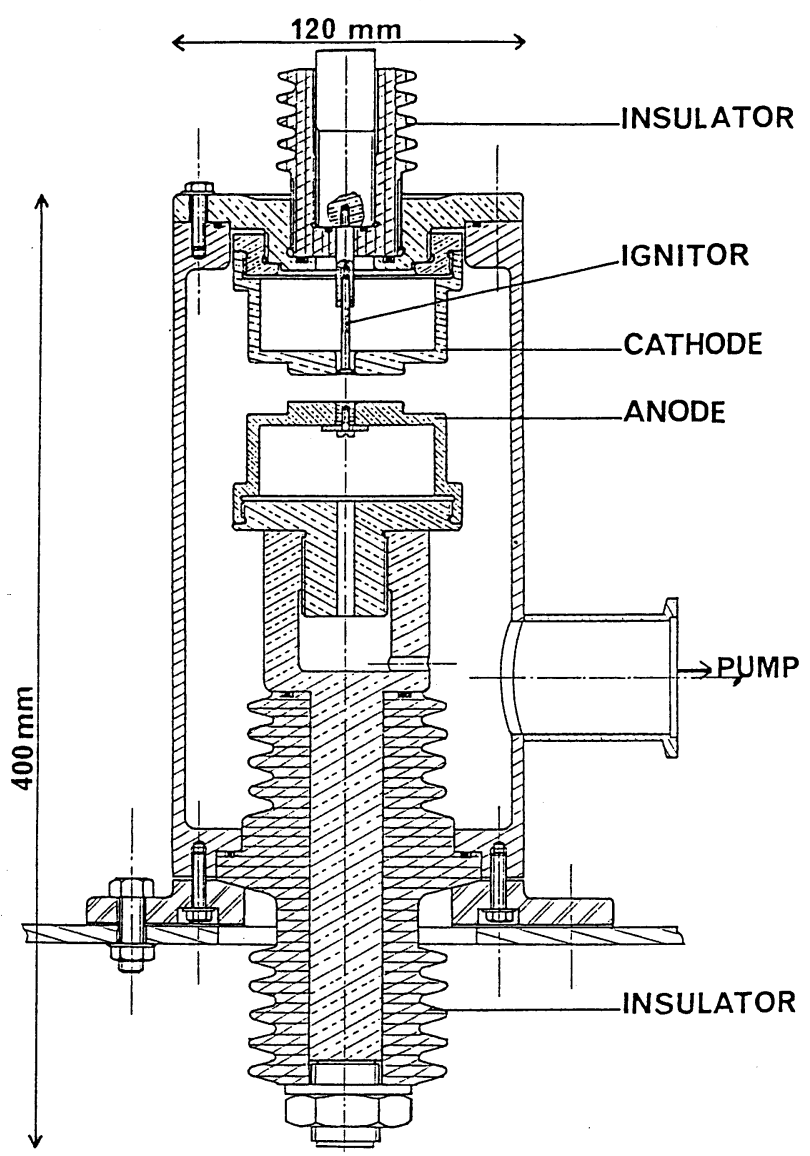

Fig. 1. - Structure du conjoncteur-disjoncteur.

[Triggered vacuum switch tube.]

2.2 LE BANC D'ESSAI. - Il est représenté sur la figure 2 et comporte deux circuits indépendants. Le premier, formé d'une inductance $L_{1}$, d'un condensateur $C_{1}$ et d'une résistance $R_{1}$ branchés en série, fournit au prototype testé une impulsion sinusoïdale

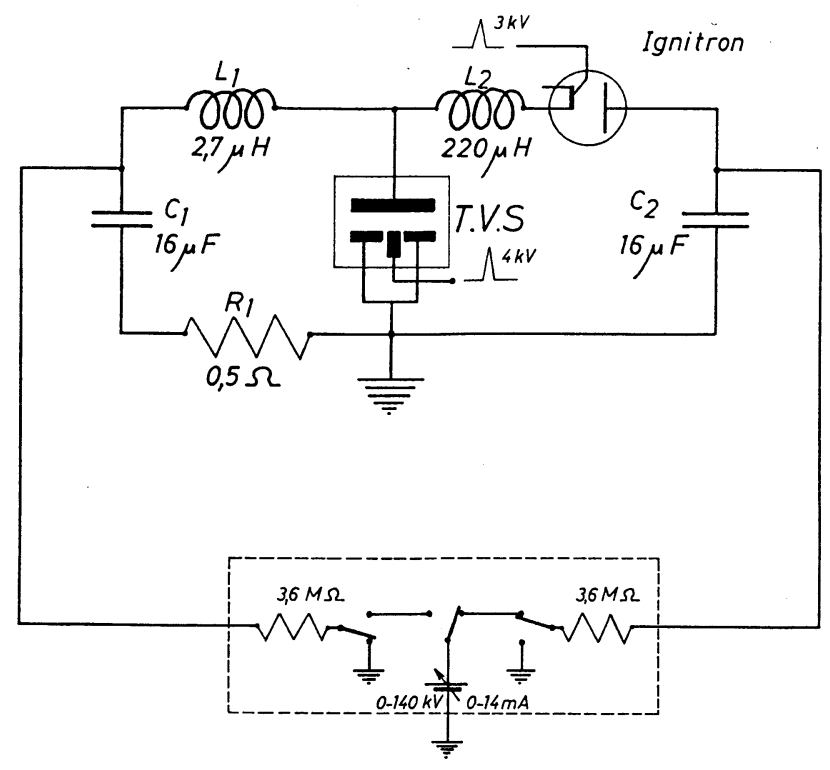

Fig. 2. - Circuit électrique du banc d'essai.

[Test circuit.]

amortie $i(t)$ d'une durée de $27 \mu$ s. L'amplitude de $i(t)$ est fonction de la tension $V_{C_{1}}$ de charge du condensateur $C_{1}$ :

$$
I_{\mathrm{p}} / V_{C_{1}}=1,3 \mathrm{~A} / \mathrm{V}
$$

Le second circuit, composé d'une inductance $L_{2}$ excédant largement $L_{1}$ et d'un condensateur $C_{2}$, assure le test en tension. L'ignitron est déclenché deux microsecondes après le passage du courant par zéro (correspondant à l'extinction naturelle du courant dans le disjoncteur). Il entraîne la décharge du condensateur $C_{2}$ dans le condensateur $C_{1}$. La tension aux bornes du disjoncteur crô̂t d'une valeur $V_{\mathrm{r}}=-0,05 V_{C_{1}}$ à une valeur $V_{\mathrm{p}}$ très proche de la tension initiale aux bornes de $C_{2}$. Nous notons que nos moyens de mesure ne nous permettent pas de déterminer la valeur de la vitesse de variation de la tension de rétablissement à l'extinction du courant. Il nous semble cependant que sa valeur dépasse les $10^{9} \mathrm{~V} / \mathrm{s}$ pour des tensions de plus de $10^{4} \mathrm{~V}$ (Fig. 3).

Les essais ont pour but de rechercher les variations du courant critique maximal admissible, au-delà duquel nous n'avons plus de réisolement, en fonction des différents paramètres géométriques et physiques. Dans une première étape les deux condensateurs sont chargés à une même tension. Le courant crête de conduction $\left(I_{\mathrm{p}}\right)$ et la tension crête de rétablissement $\left(V_{\mathrm{p}}\right)$ sont alors liés

$$
\frac{I_{\mathrm{p}}}{V_{\mathrm{p}}}=1,3 \mathrm{~A} / \mathrm{V} \text {. }
$$

Pour un lot de paramètres donnés les essais sont poursuivis jusqu'à la stabilisation des résultats. 


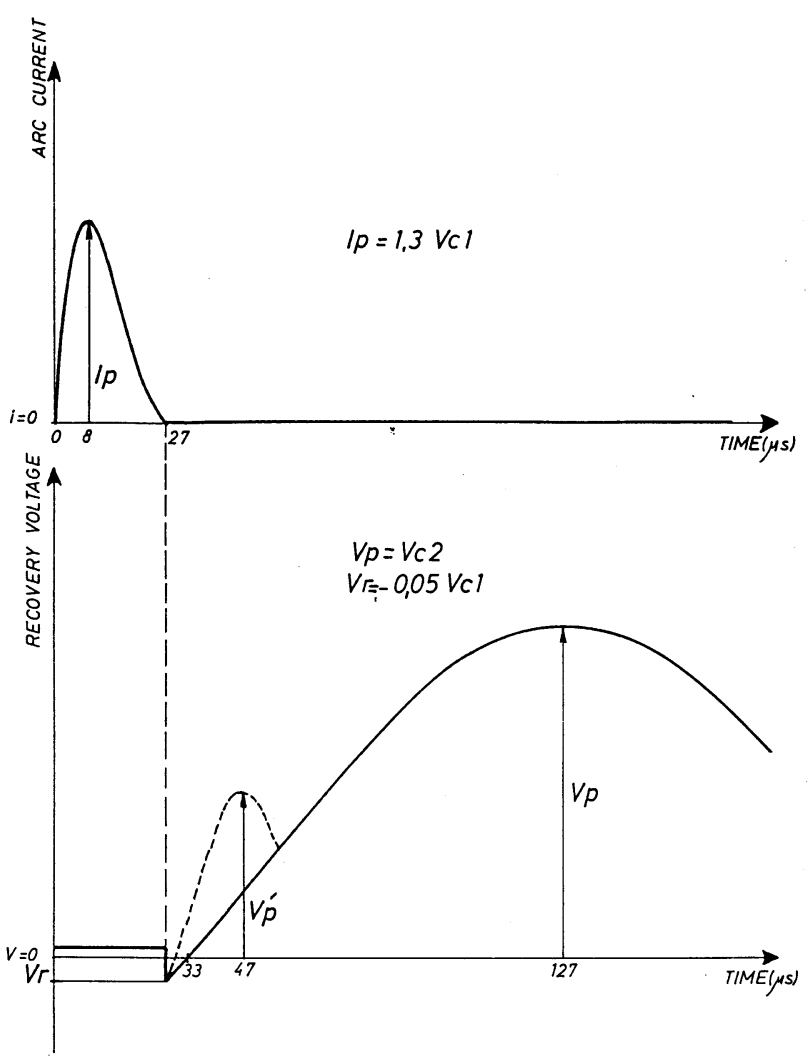

Fig. 3. - Formes d'onde du courant et de la tension.

[Current waveform and voltage waveform.]

\section{Paramètres considérés.}

Nous avons considéré, tout d'abord les paramètres géométriques et utilisé des électrodes planes et massives en cuivre OFHC de cinq diamètres différents $(22,28,38,56$ et $69 \mathrm{~mm})$. Nous avons opté pour une plage de leurs distances de séparation comprises entre 1 et $10 \mathrm{~mm}$. Nous nous sommes ensuite intéressés à des paramètres physiques (résistivité et masse spécifique) en faisant appel à d'autres matériaux tels l'Inconel et l'aluminium (diamètre $56 \mathrm{~mm})$.

De plus nous avons réalisé l'association mixte cathode en aluminium, et anode en cuivre. L'aide à la diffusion de l'arc par champ magnétique extérieur a été également testé, les électrodes dans ce cas ont une structure spécifique (le champ magnétique est proportionnel au courant d'arc).

\section{Résultats expérimentaux et discussion.}

4.1 INFLUENCE DES PARAMÈTRES GÉOMÉTRIQUES. - Nous considérons ici la situation où les condensateurs $\left(C_{1}, C_{2}\right)$ sont chargés à une même tension $\left(I_{\mathrm{p}} / V_{\mathrm{p}}=1,3 \mathrm{~A} / \mathrm{V}\right)$. La figure 4 représente les variations du courant critique au-delà duquel nous n'avons plus de réisolement, en fonction de la distance interélectrodes (paramétrées par leur diamètre).

A la constatation du passage du courant critique par un maximum, nous devons adjoindre celles relatives aux empreintes notées sur les électrodes lors du démontage. Comme nous l'avons déjà noté par ailleurs [4], la cathode est dans tous les cas, dotée d'aigrettes globalement radiales largement étalées autour de l'orifice central. Cette empreinte occupe quasiment toute la surface plane de l'électrode, sur la partie ascendante de la courbe $I_{\mathrm{c}}=f(d)$ (Fig. 4).

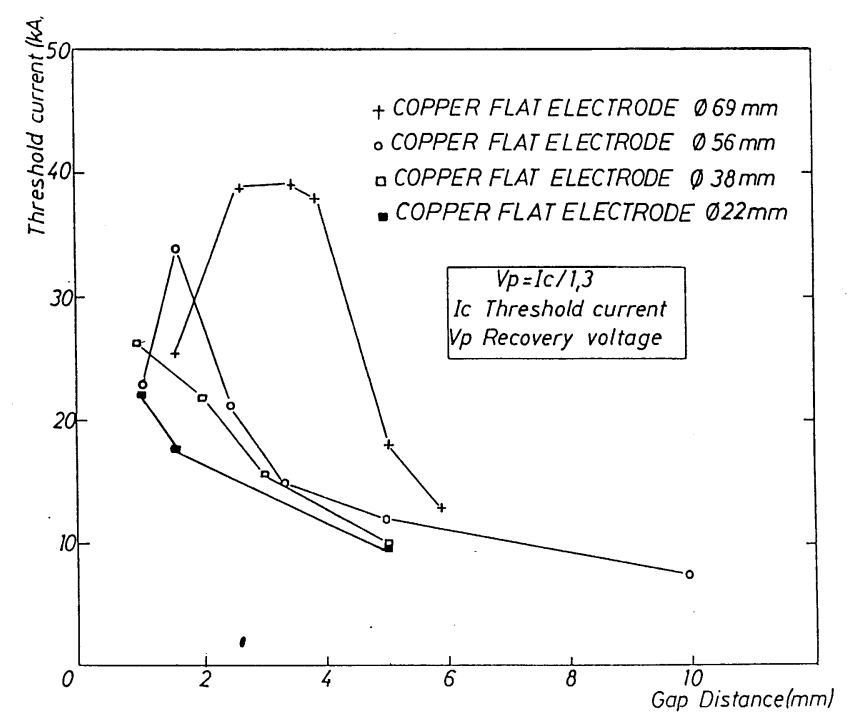

Fig. 4. - Courant critique en fonction de la distance de séparation.

[Threshold current as function of gap distance.]

L'anode par contre est dotée en son centre d'une tache circulaire bleutée de dimension plus réduite (quelques millimètres de diamètre) fonction décroissante de la distance interélectrode. Elle disparaît totalement sur toute la portion ascendante de la courbe jusqu'au courant critique maximal.

Nous constatons dans tous les cas une absence de corrosion apparente à la surface des électrodes. Une plus fine observation nous révèle que les cratères épais sur les taches bleutées ne dépassent pas $100 \mu \mathrm{m}$ (Fig. 5). Nous pouvons donc exclure l'existence de taches anodiques, telles celles notées à plus basse fréquence dans les régimes d'arcs concentrés $[6,7]$. Si, sur cette zone descendante de la courbe, nous nous intéressons à la densité apparente de courant crête, ramenée à la surface de la tache anodique bleutée sus indiquée, nous trouvons une valeur approximativement constante de $80 \mathrm{~A} / \mathrm{mm}^{2}$. Les ruptures d'isolement post conduction constatées au-delà du courant critique [4] sont semble-t-il d'origine thermique [14]. Sur ce critère, cette densité, fonction de la racine carrée de la fréquence du 


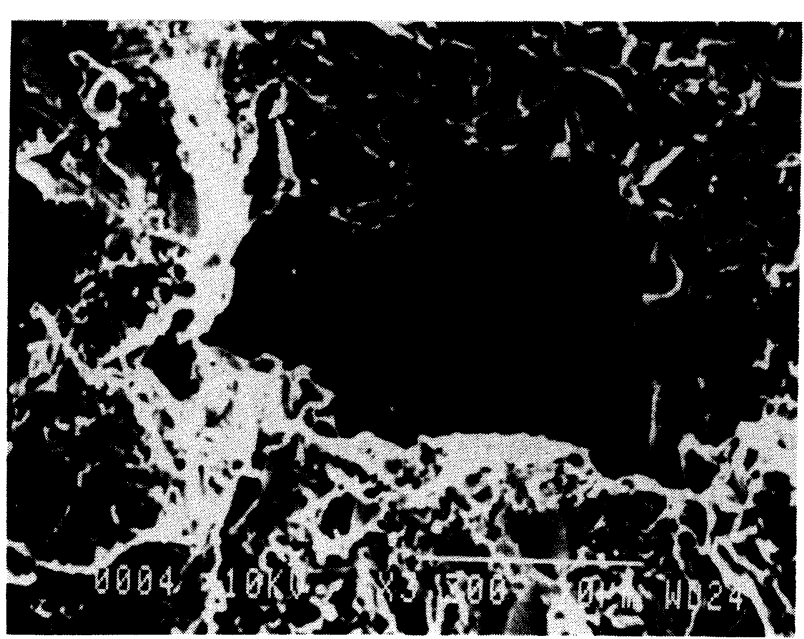

Fig. 5. - Erosion de la cathode.

[Electrode erosion (cathode).]

courant de conduction donnerait à $50 \mathrm{~Hz}$ une valeur de $4 \mathrm{~A} / \mathrm{mm}^{2}$. Cette valeur est paradoxalement très proche de celle liée à l'apparition de taches anodiques à $50 \mathrm{~Hz}$ [6].

Une observation plus globale, de cette zone descendante des courbes, intégrant le diamètre des électrodes, nous conduit à relier le courant critique au rapport diamètre $\Phi$ utile (hors arrondi) des électrodes à leur distance de séparation $d$ (Fig. 6). Nous trouvons, à l'instar des références [7] précisant à $50 \mathrm{~Hz}$ le courant critique d'apparition de taches anodiques, une relation de la forme :

$$
I_{\mathrm{c}}=\alpha\left[\frac{\Phi}{d}\right]^{m} m=0,73, \alpha=2,2 \mathrm{kA} .
$$

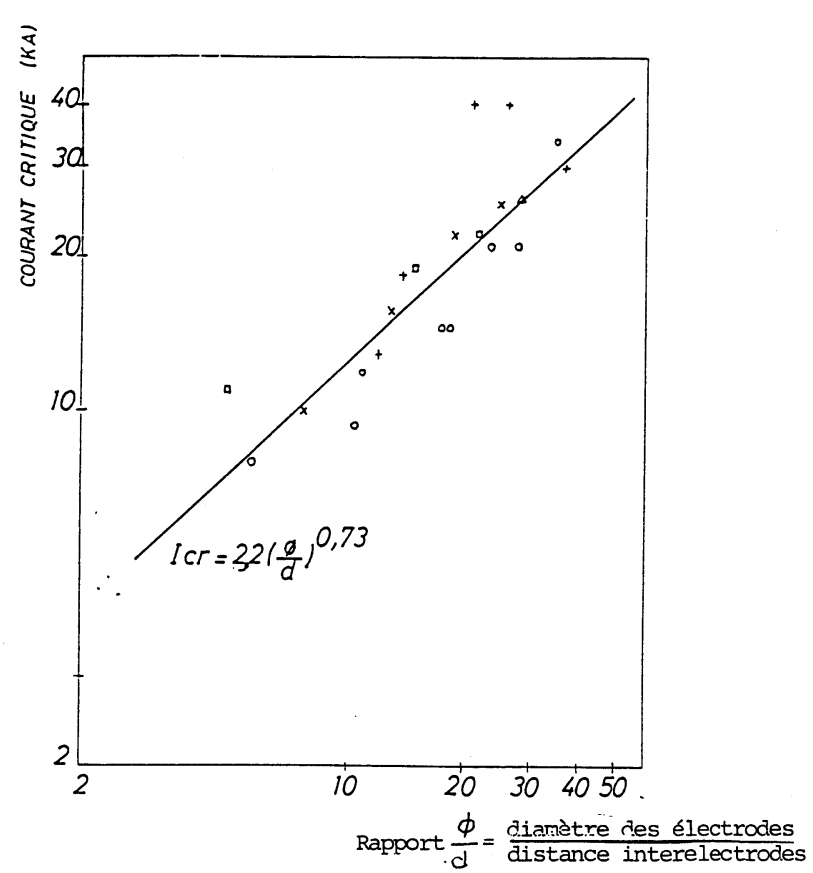

Fig. 6. - Courant critique en fonction de $(\Phi / d)$.

[Threshold current as function of $(\Phi / d)$.]
Pour les petites distances de séparation, partie ascendante de la courbe (Fig. 4) nous nous heurtons directement aux limites de tenue diélectrique du vide, malgré la situation de régime d'arc diffus, qui brident les performances du conjoncteur-disjoncteur. (Il y a quelques difficultés à assurer la charge des condensateurs).

Les valeurs maximales des courants critiques sont prises comme références sur la figure 7 , où nous avons représenté la densité apparente de courant en fonction du diamètre des électrodes. Nous retrouvons là une loi de variation de la forme :

$$
j_{\mathrm{cm}}=k \cdot \Phi^{-n} \quad n=1,6
$$

cohérente avec celles sus indiquées.

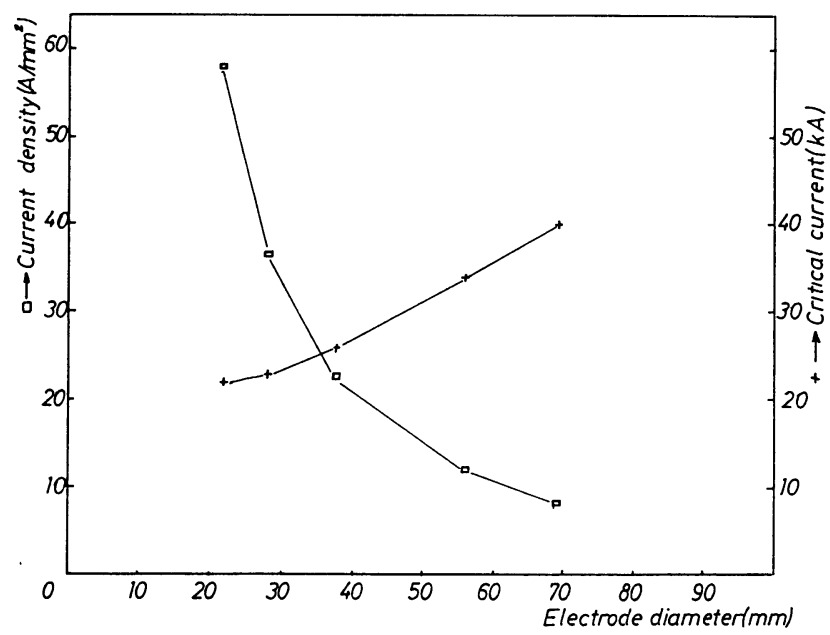

Fig. 7. - Maximums du courant critique en fonction de la distance de séparation.

[Maximum of threshold current as function of gap distance.]

Nous soulignons également que nous avons pu atteindre une puissance de commutation de $10^{9} \mathrm{~V}$. A avec des électrodes d'un diamètre de $69 \mathrm{~mm}$ (hors arrondi). Toutefois la densité de puissance référée à l'espace interélectrodes n'est que de $1,35 \times 10^{5} \mathrm{~V} . \mathrm{A} / \mathrm{mm}^{3}$ (Fig. 8). Avec des électrodes réduites $(\Phi=22 \mathrm{~mm})$ cette densité atteint une valeur de $10^{6} \mathrm{~V} . \mathrm{A} / \mathrm{mm}^{3}$ pour une puissance affichée de $0,38 \times 10^{9} \mathrm{~V}$. A.

Nous notons enfin que la vitesse de décroissance du courant à l'extinction a dépassé les $10^{9} \mathrm{~A} / \mathrm{s}$.

\subsection{INFLUENCE DE LA NATURE DES ÉLECTRODES.} - Nous avons cherché à préciser ici l'influence de la masse spécifique, et de la résistivité des matériaux des électrodes sur les performances de commutation. Dans cette optique nous avons utilisé des électrodes en aluminium puis en Inconel. 


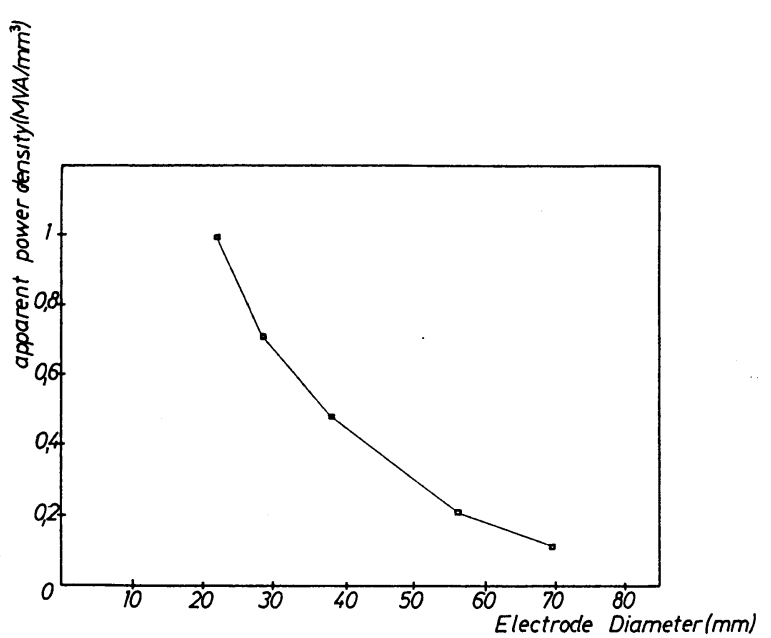

Fig. 8. - Densité apparente de puissance en fonction du diamètre des électrodes.

[Apparent power density as function of electrode diameter.]

Les résultats obtenus (Fig. 9), toujours dans les mêmes conditions que précédemment, sont visualisées sur la figure 10. L'aluminium, malgré sa faible masse atomique, est ici décevant. La faible température de fusion de ce matériau laisse penser à quelques défaillances thermiques au niveau de l'anode malgré l'empreinte diffuse, observée au démontage, au niveau de la cathode. Il apparaît effectivement qu'avec une cathode en aluminium et une anode en cuivre, les résultats sont sensiblement supérieurs à ceux obtenus avec une paire d'électrodes en cuivre. Ces résultats vont dans le sens de ceux relatés par Miller [6] qui montrent l'incidence de la nature de l'anode sur le niveau du courant critique d'apparition de spots anodiques.

Il y a tout lieu de penser que la faible température des spots cathodiques et la faible masse du matériau, impliquent une plus faible énergie des particules incidentes sur la cible anodique [6].

Les électrodes en Inconel donnent accès à des performances de commutation similaires à celles obtenues avec les électrodes mixtes aluminium (cathode) cuivre (anode).

Nous sommes conduits ici, à faire un rapprochement avec les résultats obtenus par ailleurs $[8,9]$ et dans un autre contexte, $(60 \mathrm{~Hz})$, qui mettent en relief l'apport bénéfique du chrome dans les électrodes en cuivre (alliage optimal $20 \% \mathrm{Cr} 80 \% \mathrm{Cu}$ ) sur les performances des disjoncteurs à vide. L'Inconel contient une quantité de chrome assez proche (14 à $17 \%$ ) pour il est vrai $72 \%$ de nickel.

4.3 INFLUENCE D'UN CHAMP MAGNÉTIQUE. L'empreinte anodique bleutée notée plus haut étant un signe manifeste d'une striction de l'arc sur l'anode lors de son développement, nous avons

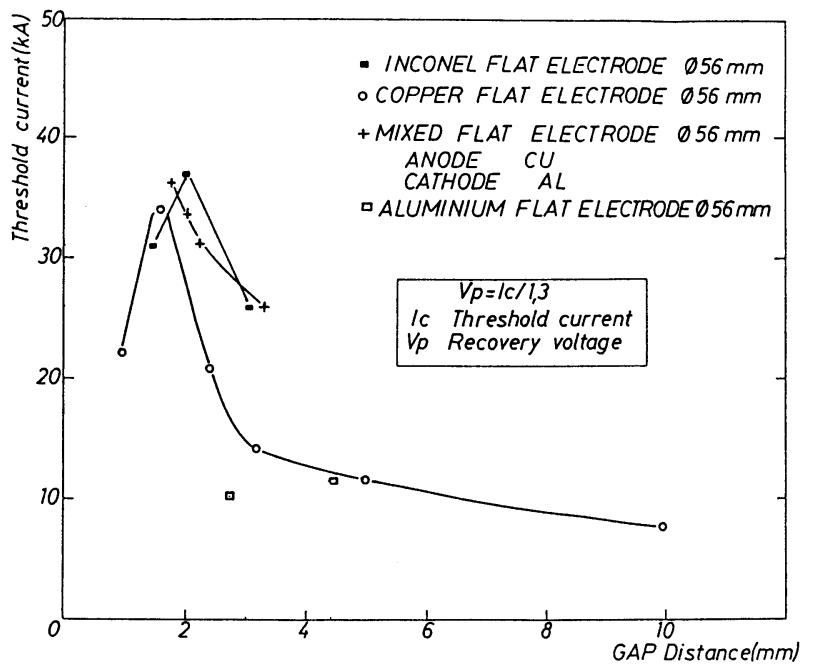

Fig. 9. - Courant critique en fonction de la distance de séparation pour différents matériaux.

[Threshold current as function of gap distance for different electrode materials.]

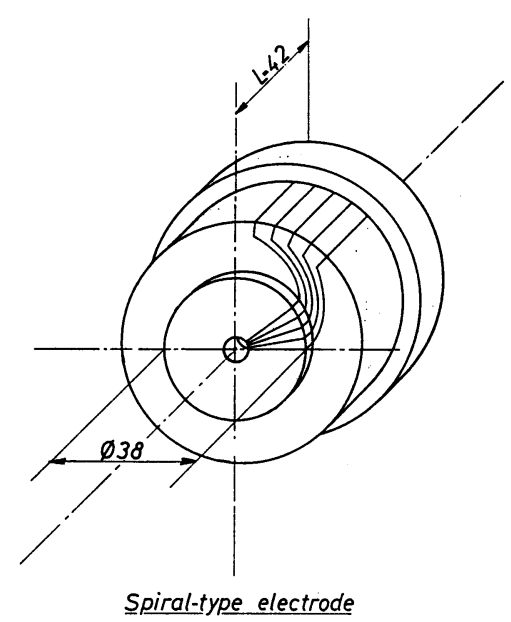

Fig. 10. - Structure de l'électrode à spirales.

[A spiral type electrode.]

cherrché quelques améliorations de la diffusion de l'arc en suscitant son interaction avec un champ magnétique axial, via des électrodes adaptées. Nous nous sommes inspirés des travaux réalisés sur la disjonction sous vide à basse fréquence $[4,7,10]$.

4.3.1 Electrodes spiralées multifentes. - Un champ magnétique axial est généré dans l'espace interélectrodes, en imposant une rotation du courant d'arc avant son arrivée sur la surface active des électrodes (Fig. 10). Ce champ est proportionnel au courant d'arc et estimé ici à $5-10 \mathrm{mT} / \mathrm{kA}$. Les résultats obtenus explicitent son influence mais demeurent sur le plan commutation fort décevants (Fig. 11). 


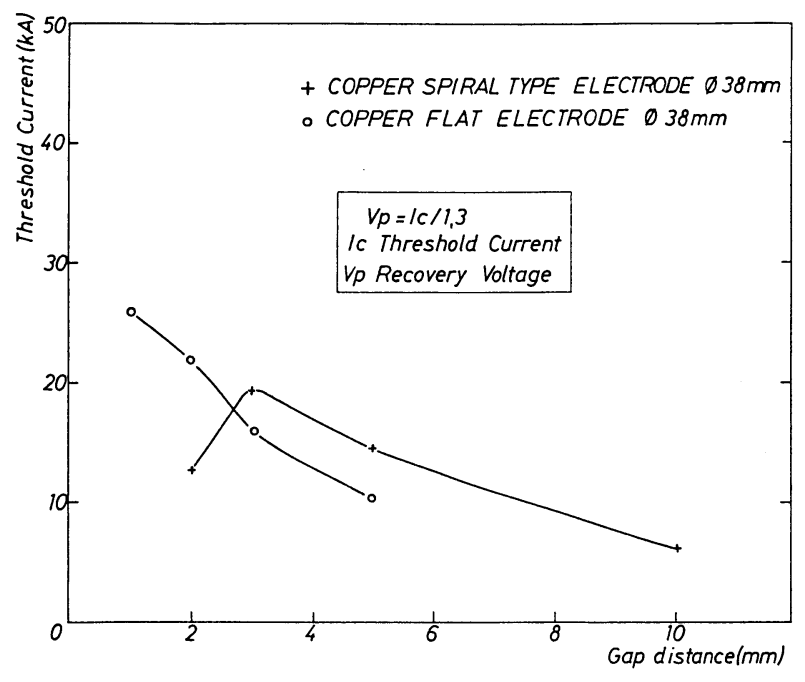

Fig. 11. - Comparaison des résultats des électrodes lisses et des électrodes à spirales.

[Comparison between flat electrode and spiral type electrode.]

Nous constatons un accroissement des empreintes bleutées notées précédemment. Il semble que les arêtes liées aux fentes permettant le passage du champ magnétique sur la face active suscitent la fixation de lignes de courant d'arc. Une observation fine au microscope électronique à balayage, fait état d'une érosion marquée en ces lieux; (cratères de quelques dizaines de micromètres) (Fig. 12).

Pour pallier cet inconvénient, nous avons mis à profit la grande résistivité de l'Inconel, pour élaborer des électrodes présentant une surface active sans aspérité et "perméable » au champ magnétique [7].

4.3.2 Surface active "perméable » au champ magnétique. - La surface active est ici réalisée par une

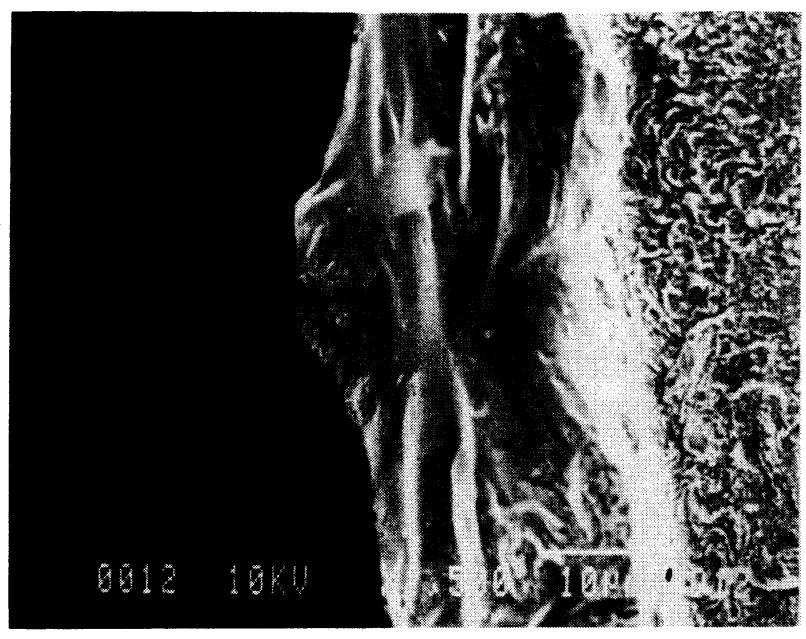

Fig. 12. - Erosion le long d'une arête d'une électrode à spirales.

[Erosion of a slit line.]

plaque mince en Inconel de $1 \mathrm{~mm}$ fixée sur le support creux en cuivre qui impose la rotation du courant avant accès à l'arc.

a) L'anode et la cathode imposent le champ magnétique (Fig. 13a) :

la plaque en Inconel apporte une atténuation au champ magnétique de l'ordre de $1 \%$ [14] pour laisser place à un champ axial interélectrode compris entre 5 et $8 \mathrm{mT} / \mathrm{kA}$ (Fig. 14a). Les résultats obtenus sont décevants (Fig. 15). Ils nous ont incités à remplacer l'anode par une électrode massive en Inconel.

b) Anode massive en Inconel, cathode génératrice du champ magnétique (Fig. 13b) :

dans ce cas le champ magnétique est dans l'espace interélectrode fortement radial (Fig. 14b). Il
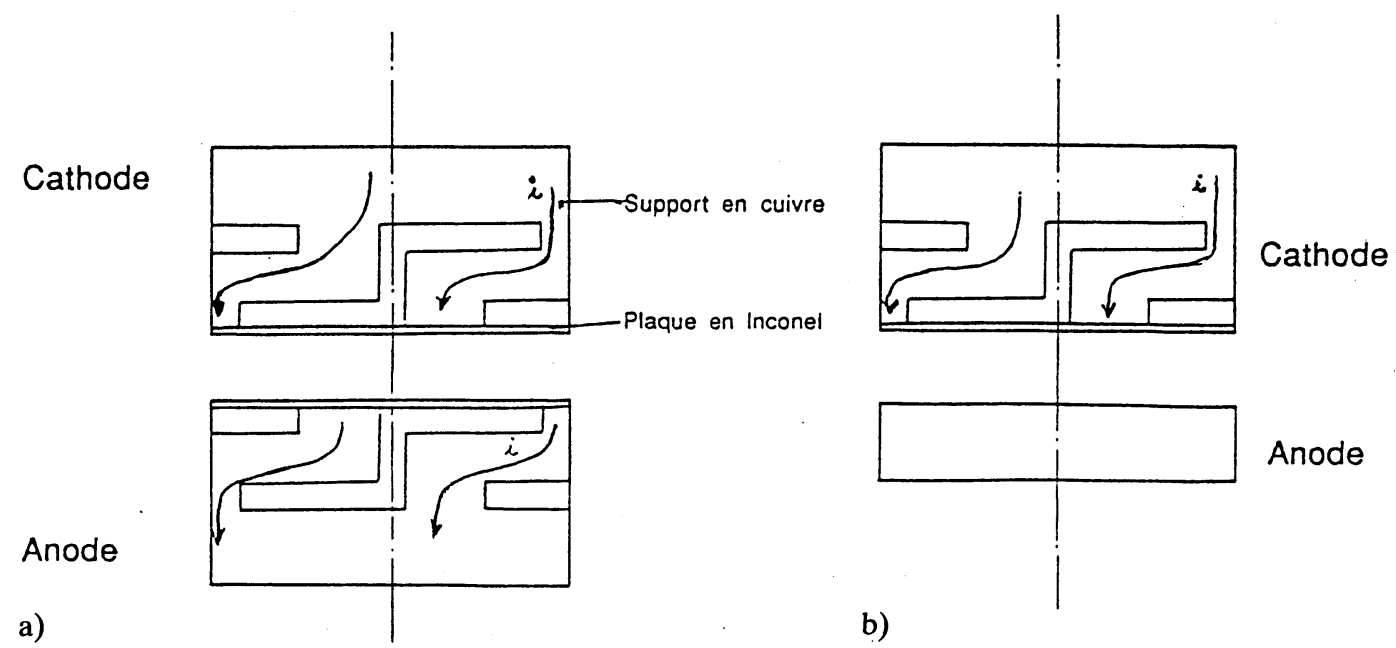

Fig. 13. - Structure des électrodes à champ magnétique.

[Magnetic field electrode structure.] 


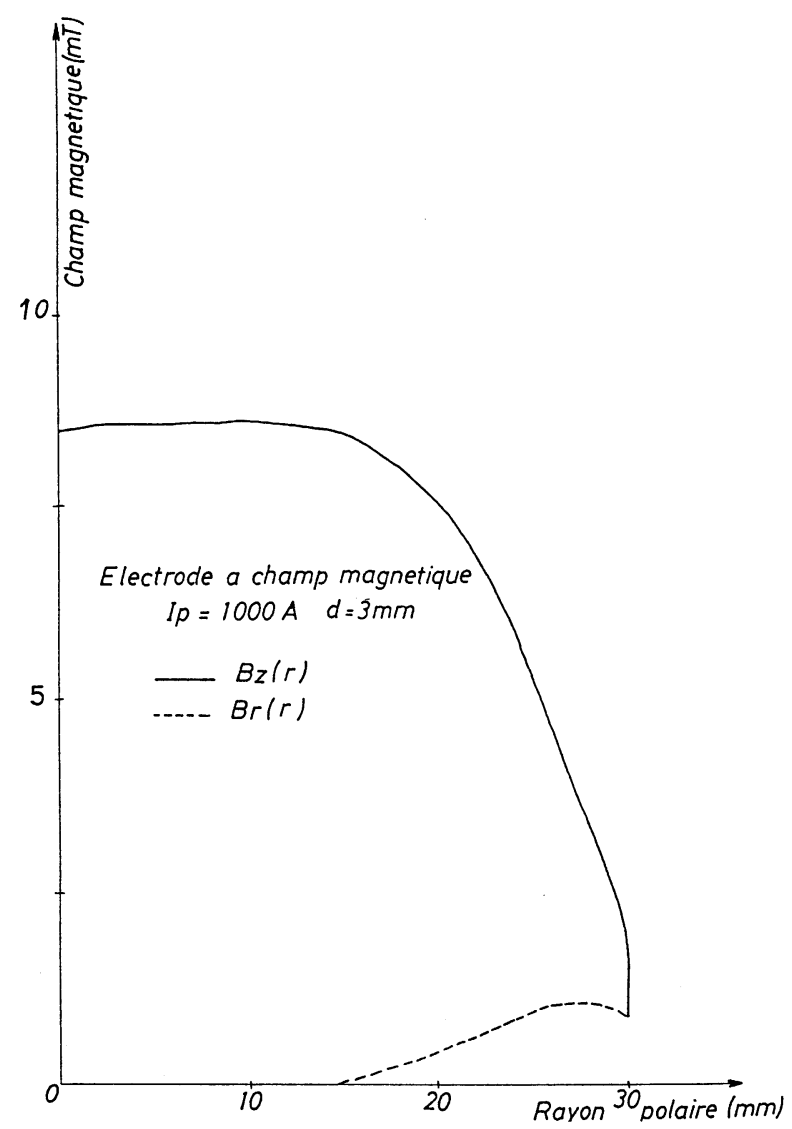

a)

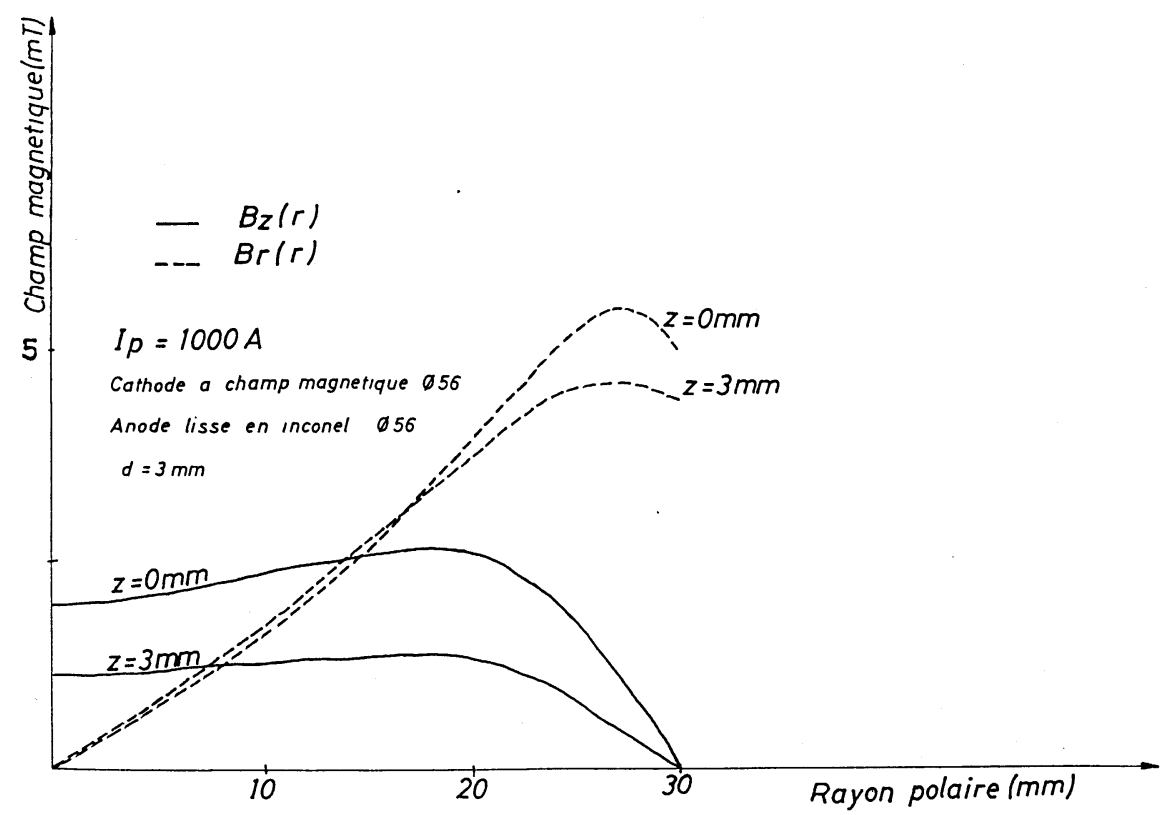

b)

Fig. 14. - Distribution du champ magnétique.

[Magnetic field distribution.]

s'oppose à la striction naturelle de l'arc côté anode, et pousse à sa diffusion périphérique, voire son instabilité. Les performances optimales de disjonction sont ici sensiblement supérieures à celles obte- nues avec les électrodes massives (plus de $15 \%$ ) (Fig. 15).

Il est possible que le champ magnétique axial mis en jeu dans la première série d'essais (cas de la 


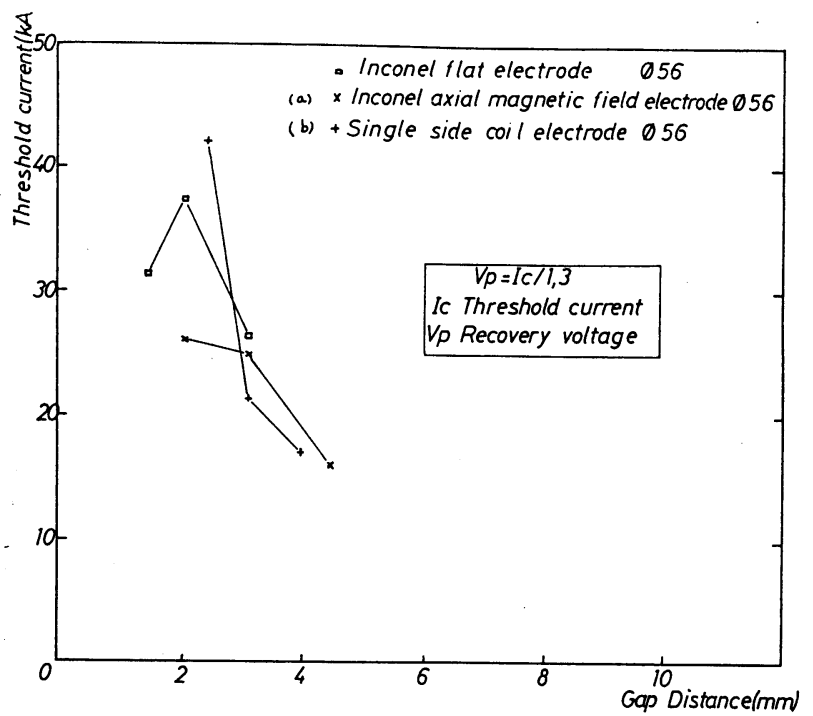

Fig. 15. - Influence d'un champ magnétique.

[Effect of a magnetic field.]

Fig. 13a) soit insuffisant. Un accroissement de la composante radiale du champ magnétique est à même de compenser cette défaillance, il est vrai de manière plus aigüe.

\section{Optimisation de la puissance de commutation.}

Nous avons utilisé jusqu'à présent une liaison directe entre le courant crête de conduction et la tension crête de test de recouvrement diélectrique :

$$
\frac{I_{\mathrm{p}}}{V_{\mathrm{p}}}=1,3 \mathrm{~A} / \mathrm{V} \text {. }
$$

Afin de modifier ce rapport, les deux circuits correspondants sont découplés. Il est ainsi possible de chargèr les deux blocs de condensateurs à deux tensions différentes. Nous avons pu ainsi, pour un lot de paramètres donné, réduire le courant de conduction du conjoncteur-disjoncteur $\left(I_{\mathrm{p}}<I_{\mathrm{c}}\right)$ et préciser le gain en tension crête lors du recouvrement diélectrique (Fig. 16). Il apparaît qu'un léger écart du courant critique noté précédemment, apporte un substanciel gain dans la vitesse de recouvrement diélectrique. Les limites du banc d'essai ne nous ont pas permis de préciser les puissances optimales de commutation.

\begin{tabular}{|c|c|c|c|c|c|c|}
\hline Electrodes & \multicolumn{2}{|c|}{$\begin{array}{c}\text { Aluminium } \\
\emptyset=56 \mathrm{~mm} \quad \mathrm{~d}=2,8 \mathrm{~mm}\end{array}$} & \multicolumn{2}{|c|}{ 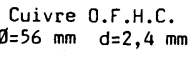 } & \multicolumn{2}{|c|}{$\begin{array}{l}\text { Cuivre 0.F.H.C. } \\
\emptyset=69 \mathrm{~mm} \quad \mathrm{~d}=3,8 \mathrm{~mm}\end{array}$} \\
\hline$I_{c}(k A)$ & 11 & 10 & 21 & 20 & 38 & 36 \\
\hline$v_{c}(k v)$ & 8 & 23 & 16 & 30 & 29 & $>44$ \\
\hline
\end{tabular}

Fig. 16. - Relation entre courant de conduction et tension de réisolement.

[Relationship between current and recovery voltage.]

Ces résultats sont à rapprocher de ceux obtenus par Zalucki avec des électrodes èn alliage cuivre chrome à des fréquences proches des nôtres [12], ou encore par Lindmayer et Unger-Weber à 50 Hz [13].

\section{Conclusion.}

Nous avons présenté une application non classique de la coupure sous vide. Nous avons étudié le développement et l'extinction rapide $\left(27 \times 10^{-6} \mathrm{~s}\right)$ d'un arc électrique dans le vide. Nous avons pu retrouver un régime d'arc diffus et préciser quelques conditions géométriques et physiques permettant de le préserver au-delà de quelques $10^{4} \mathrm{~A}$. Les puissances de coupure atteintes excèdent alors $10^{9} \mathrm{~V}$.A (électrodes en $\mathrm{Cu}$, de $69 \mathrm{~mm}$ de diamètre). Cette puissance ramenée au volume d'espaces interélectrodes est alors $0,14 \times 10^{6} \mathrm{~V}$.A $/ \mathrm{mm}^{3}$ pour les électrodes $\Phi 69 \mathrm{~mm}$, mais peut atteindre avec des électrodes plus petites ( $\Phi$ 22) une valeur de $10^{6} \mathrm{~V} . \mathrm{A} / \mathrm{mm}^{3}$. Une optimisation de fonctionnement du système (réduction du courant de conduction) est semble-t-il à même d'améliorer notablement ces performances.

Lors d'études complémentaires, le temps de remontée de la tension testant le recouvrement diélectrique (Fig. 3), a pu être réduit par réduction de l'inductance $L_{2}$ jusqu'à $20 \times 10^{-6} \mathrm{~s}$ (limite du banc d'essai) sans que nous notions une modification des résultats principaux exposés. Tout comme la puissance de coupure, le paramètre temps de commutation mérité une grande attention: des temps de quelques $10^{-6} \mathrm{~s}$ sont d'ores et déjà recherchés.

Les performances et la rapidité de fonctionnement d'un tel conjoncteur-disjoncteur laissent entrevoir quelques sérieuses perspectives dans le transfert inductif d'énergie $[14,15]$ en électrotechnique impulsionnelle.
[1] Weldon W. F., IEEE Spect. (1985) pp. 59-66.

[2] Giorgi D., LoNDER K., LONG J., NAVAPANiSH T. and ZuCKer O., Proceeding of the 5th IEEE Pulsed Power Conference (1985) pp. 615-618.

[3] Allano S., Delmas A. and Rioux C., Proceeding of the 6th IEEE Pulsed Power Conference (1987) pp. 449-452.

[4] Delmas A., Dongfack A. et Rioux C., Revue Phys. Appl. 22 (1987) 761-765.

[5] Reece M. P., Proc. IEE 110 (1963) 796-811. 
[6] Miller H. C., Rapport interne G.E. (1988).

[7] Frind G., Caroll J. J., Goody C. P. and Tuotty E. J., IEEE PAS 101 (1982) 775-782.

[8] YanABu S. et al., IEEE PS 15 (1987) 524-531.

[9] YANABU S. et al., $13^{\mathrm{e}}$ Symposium international sur les décharges et l'isolement électrique dans le vide, Paris (1988) pp. 131-137.

[10] YanABU S. et al., IEEE PAS 104 (1987) 166-172.

[11] OKAWA O. et al., IEEE PS 15 (1987) 533-537.

[12] ZALUCKI Z., 13e Symposium international sur les décharges et l'isolement électrique dans le vide, Paris (1988) communication non publiée.
[13] Lindmayer M. and UNGer-Weber F., 13e Symposium international sur les décharges et l'isolement électrique dans le vide, Paris (1988) pp. 330-334.

[14] HAdDAd N., Etude de la conjonction-disjonction sous vide applicable aux transferts rapides d'énergie, Thèse de Doctorat de l'Université de Paris 6 (1989).

[15] Bauville G., Delmas A., Haddad N. et RiouX C., $13^{\mathrm{e}}$ Symposium international sur les décharges et l'isolement électrique dans le vide, Paris (1988) pp. 404-406. 